\title{
Broad donor consent for human biobanks in Germany and Europe: a strategy to facilitate cross-border sharing and exchange of human biological materials and related data
}

https://doi.org/10.1515/labmed-2017-0064

Received May 23, 2017; accepted December 9, 2018; previously published online March 9, 2019

Brief summary: Human biological materials stored long term in biobanks are increasingly recognized as fundamental resources for biomedical research progress. Regarding the openness of future medical questions, the Working Party of the German Medical Ethics Committees has recently updated its broad consent template as one key-element of a framework permitting almost unlimited storage and unrestricted use of human biological samples and related data for biomedical research.

\section{Abstract}

Background: Human biobanks are generally recognized as essential resources for effective biomedical research. All over the world biosamples and data from human subjects are collected in large biobanks. The biological material is stored long term for current and future (undetermined) research issues, which often require cross-border exchange of biosamples and related data.

Content: Commonly, the informed consent for research on human biospecimen is intended to cover only defined, specific research objectives. In June 2016, the biobank

*Correspondence: Univ.-Prof. Dr. med. Roland Jahns, Interdisciplinary Bank of Biomaterials and Data Würzburg (ibdw), University and University Hospital of Würzburg, Building A8/A9, Straubmühlweg 2a, 97078 Würzburg, Germany,

E-Mail: jahns_r@ukw.de

Jörg Geiger: Interdisciplinary Bank of Biomaterials and Data Würzburg (ibdw), University and University Hospital of Würzburg, Würzburg, Germany

Irene Schlünder: Technology, Methods, and Infrastructure for Networked Medical Research (TMF), Legal Affairs and Bio-Ethics, Berlin, Germany

Daniel Strech: Charité - University Medicine Berlin, Institute of Health Research (BIG/BIH) QUEST-Center, Berlin, Germany Matthias Brumhard: Clinical Ethics Advisor, Section 11 Medicine, Justus-Liebig-University Gießen, Gießen, Germany Sebastian Graf von Kielmansegg: Chair for Public and Medical Law, Christian-Albrechts-University Kiel, Kiel, Germany
Task-Force of the Working Party of the German Medical Ethics Committees (WP-GMEC) updated its template for the broad use of human biological samples and related data. It complies with the current Organisation for Economic Co-operation and Development (OECD) and World Medical Association (WMA) recommendations and furnishes a framework that permits long-term storage and multi-purpose research use of human biological material and related data, including cross-border research.

However, both (i) human biobanks storing and (ii) research projects requesting "broad consent" biological samples generally require an ethical approval; in addition, "broad consent" conditions should be reciprocated by making biobank processes transparent and by fostering both donor and public involvement.

Outlook: The broad consent template of the WP-GMEC clearly states that biological samples and data donated for medical research serve to address current and future research questions. It appears perfectly suited as a template for a Europe-wide harmonized broad consent facilitating biobank-based cross-border research.

Keywords: broad consent; cross-border exchange; donated biological material; independent competent ethics committees; stakeholder involvement.

\section{Introduction}

Today, human biobanks have been recognized as important resources for medical research, in particular for the progress in targeted drug therapy [1, 2]. A "biobank" in the sense of the present article collects and stores human biological material and related data for often not foreseeable medical research purposes [3-8]. Such research may comprise basic research as well as applied (clinical) research directed toward (i) a faster and more precise detection of diseases (diagnostics), (ii) a prediction of disease development and outcomes (prognosis), (iii) a more precise and efficient treatment (therapy) and/or (iv) avoiding factors/ conditions resulting in (prevention of disease occurrence) 
or accelerating disease development (prevention of disease progression).

The majority of modern human biobanks currently (or will in the near future) strive for inter- or multidisciplinary co-operations and networking between single or multiple collections of human biological material with established governance and procedural rules, including access and sharing policies $[1,3,9]$. In the past two decades, such policies have been developed and successfully implemented in a variety of German populationbased (KORA, SHIP, NAKO [10-14]) and - more recently - also in disease-oriented research networks (e.g. GANI MED, DKTK, DZL, DZHK [15-18]). With regard to sample access and sharing, German biobanks are bound to European, national and state laws. As a basic principle, irrespective of the site where biological samples and related data are ultimately used, the respective national law is applicable to the collection and processing of samples and data [19]. Current and future (not foreseeable) research issues often require cross-border exchange of biological samples and related data, but such processing of data and use of biospecimen must be compliant with the provisions of the donor's consent (and/or - in exceptional cases - with the decision of an "independent competent ethics committee" [for definition, please see text box]) [20]. On this background, many of the internationally prevailing informed consents are inadequate because of their limitations to specific diseases or research objectives.

Hence, an expanded and - at the same time - crossborder-oriented scope is required, balancing individual donor rights and research interests [21]. Based on previous "best practice" models and the experiences with consent management and consenting procedures gathered in previous larger population-based studies (KORA, SHIP, GANIMED [10-12]), the biobank task force of the permanent Working Party of the German Medical Ethics Committees (WP-GMEC) has developed in 2013 [22] and recently updated [23] its master template for the "broad use of human biological material and related data for medical research". The document focuses on large interdisciplinary clinical biobanks (associated, e.g. to university hospitals) and has been re-approved by the general assembly of all independent competent German medical ethics committees (see the following text box for definition) in June 2016.

\section{Definition}

The term independent "competent" ethics committees is specific for Germany and represents the generally accepted expression for currently 52 "public" ethics committees established based on federal state law (Landesrecht) situated at 36 German Universities (with Medical Faculties), and at each (federal) state's Physicians Chamber (15 Landesärztekammern), and three (federal) state's ethics committees (Länder Berlin, Bremen, and Sachsen-Anhalt). Competent ethics committees by definition must be independent, interdisciplinary, and following EU-regulation 536/2014 (adopted by Germany on December 20, 2016), as a minimum standard, must be composed of at least:

(a) 1 lawyer/judge,

(b) 1 ethicist,

(c) 1 biometrician/statistician,

(d) 3 clinically experienced physicians from different clinical disciplines, including one pharmacologist or toxicologist, and

(e) 1 lay member.

Competent ethics committees in Germany are different from private ethics committees (e.g. the "Freiburger Ethikkommission") or clinical ethics committees required in certain situations with critically ill patients in order to guide the treating physicians. Because competent ethics committees are installed by law and relevant stakeholders in the approval procedure for clinical phase I-III trials according to Medicines Law or Medicines Product Law, they are generally more powerful and often have more interdisciplinary and biometrical/ statistical competence and experience than the internationally prevailing research ethics committees, the said "private ethics committees", "clinical ethics committees", "institutional research (ethics) committees", "ethical and/or scientific review boards" (e.g. companies, biobanks from the pharmaceutical industry), and also differ from any "use and access committees" at biobanks and/or data warehouses.

The updated template acknowledges international requirements for consent in (human) biobank research $[7,24]$ and particularly fulfils the current Organisation for Economic Co-operation and Development (OECD) [25-27] and World Medical Association (WMA) recommendations [20] for biobanks. On this basis, it furnishes a framework that enables long-term storage and multipurpose use of human biological material and related data, provided they are exclusively employed in biomedical research, including cross-border research. The following sections will serve to present this framework more in detail.

\section{Ethical framework for a broad consent}

From the perspective of a potential donor, the collection and storage of human biological material for medical research purposes always requires a clearly assigned purpose on an individual basis [21]. Consequently, the purpose of the collection, storage and intended use of human biological material and related data by a biobank 
should be specified as exactly as possible [28]. Such purposes might be, e.g. the conduct of a particular clinical study, or research focused on a specific disease (i.e. lung cancer) or well-defined disease entities (i.e. cardiovascular diseases or brain disorders).

On the other hand, biobanks must be prepared to satisfy future medical research objectives and meet future challenges in public health by permitting broad use of human biological material and related data, including cross-border exchange $[8,9,19,28]$. The ethical framework should enable biobanks to fulfil their mission in (i) opening new vistas for medical research and (ii) supporting optimization of public health care. However, as a pre-condition for legal validity of a broad consent, the unpredictability of the future use of donated biological samples and related data must be compensated by appropriate measures and procedures $[3,4,8,9,21,25,26]$. In this regard, in Germany, independent competent ethics committees are of paramount relevance for both (i) the assessment of the human biobank itself (during set-up and during operation) and (ii) the assessment of individual research projects requesting "broad consent" biological samples as a general pre-condition for the release and delivery of such samples [8, 28, 29].

When asked for broad consent, the donor has to be informed unambiguously on the broad scope of the future use of his/her biological material and related data including the option of cross-border medical research. However, even under the conditions of "broad consent" the donor should be given, at least to some extent, the opportunity to exclude certain research fields and/ or procedures from future use $[21,28]$ (similar but not identical to a so-called dynamic consent [30]). Finally, it should be noted that the "broad consent" proposed here is not unlimited but clearly restricted to biomedical research.

\section{The tasks of ethics committees regarding human biobanks}

\section{Approval of the human biobank itself}

Irrespective of legal obligations, from an ethical point of view, the establishment of a research-oriented human biobank by public or private organizations/institutions (including pharmaceutical industry) in Germany generally requires assessment by an independent competent ethics committee. The same applies in case of relevant changes of the scope or the legal owner of a biobank, or in case of the transfer of human biological material into the responsibility of another organization $[8,21,28,29]$.

For the approval of a research-related human biobank set-up and/or operated by public or private organizations/ institutions, the following documents need to be assessed [28]:

- information on the scope, governance (e.g. by-laws, user manual), procedures, means of documentation and financial plan (sustainability) of the biobank;

- information on the nature and principles of the collection, and the mode (temperature range, type of repository) and duration of storage;

- measures implemented for quality management (QM) and quality control (QC) (quality manual), intended use and security measures regarding human biological material and related data, including the management of incidental or unsolicited findings and, in case a feedback of research results to the donors is foreseen, the workflow for communicating the information to the donors;

- information documents for donors (patients/participants) and corresponding (broad) consent form(s).

\section{Approval of applying research projects}

Prior to the release and delivery of "broad consent" human biological material and/or related data for a specific biomedical research project, the human biobank must check whether the research proposal corresponding to the request has been approved by an independent competent ethics committee $[21,28,29]$, at least where such a vote is mandatory (e.g. required by the code of professional conduct [physicians] [31] and/or the Declaration of Helsinki in its current version [32]). Depending on national specifics, the respective legislation or the type and scope of the intended research project, an access committee may substitute for an independent competent ethics committee [33]. In Germany, for example, this would be (i) in case of (retrospective) biomedical research with anonymized biological material and related data or (ii) in case of a strictly internal use of patient data or biological material by the attending physician/department for quality assessment or control (depending on the respective state's hospital law).

Nevertheless, the respective German human biobank may at any time consult the local independent competent ethics committee in order to assess the research project applying for samples on its own discretion $[28,29]$. 


\section{Information documents and (broad) consent form for biobank donors}

Besides public engagement and transparent processes of a biobank, one of the essential pre-conditions for the acquisition and storage of human biological material for multiple medical research purposes is the informed (broad) consent form, signed by the donor after comprehensive written and oral consultation [28]. When approached for biobank participation, many individuals/patients want to make an active and informed choice, but for altruistic reasons are also prepared and willing to consent broadly to future research use [34, 35]. The content of the information document should be easy to understand and as concise as possible (but at the same time as comprehensive as possible). The WP-GMEC text template "for the use of human biological material and related data in biobanks" in its current version represents a first (nation-wide harmonized) step in this direction and should thus serve as a model for all German human biobanks [23, 36]. If genetic analyses of human biological material are intended, this should be specified in the information and consent documents and addressed during oral consultation.

If foreseen, the predetermined procedures for feedback of incidental or unsolicited genetical (research [!]) findings should be communicated to the donor as well. Following the current version of the Declaration of Helsinki [32] (10/2013, §26), referred to also in the current WMA recommendations [20] (10/2016, §11), information and consultation of donors should be performed by the physician or another appropriately qualified individual (personnel) trained in biobanking, able to provide supplementary information and to answer questions pertaining to the consent.

The signed (both by donor and informing physician/ qualified personnel) consent forms must be stored by the institution/hospital that has obtained the consent (or by a trusted third party [TTP], exceptionally by trusted biobank personnel [e.g. biobank office, secretary], having no access to any donor-related clinical data). The retention period of consent forms should last at least for the time period the biological material and related data will be stored and/or used for medical research purposes. A copy of the signed (broad) consent form and the information document must be handed to the donor [28].

The consent may also be obtained for long-term storage and multi-purpose use of the donor's biological material and related data for medical research, as long as this has been explicitly stated in the broad consent form and during (oral) consultation. In any case, all permissions expire with the withdrawal of the consent by the donor $[21,28]$.
When the scope and intended purpose of a human biobank or any relevant conditions under which the consent has previously been obtained change, renewal of the consent is required - except if

(i) a disproportionate effort to re-contact the respective donors has been made (e.g. the donor relocated and even with the assistance of the respective registration office(s) - the new address cannot be established, or in case the donor is deceased and neither heirs nor family members can be identified, even with the aid of the appropriate authorities), and

(ii) the scientific interest and/or potential medical benefit of such changes outweigh the interests of the donor or any other person concerned $[8,9,21,28]$.

In all such cases, it is, however, recommended to obtain additional approval by an independent competent ethics committee - at least in Germany [28].

Already existing biological material and related data available from a deceased individual may be transferred into and stored in a human biobank only, if such material and related data are fully anonymized, or if the scientific interests outweigh the vested interests of the respective individual or any other (related) person concerned. In all such cases, again, approval by an independent competent ethics committee is strongly recommended [21]. Inherently it is virtually impossible to achieve full anonymization, particularly with regard to genetic information (e.g. of biological samples containing DNA) [37]. Usually, anonymization means that the connection between a pseudonym and the personally identifiable information of the donor/patient is deleted. If the donor/patient requests destruction of his/her biological material and deletion of all related data, the anonymization procedure must be carried out only after destruction of the biological material. Nonetheless, data cannot be removed from the analyses of already completed studies and/or published research results.

It should be noted, however, that any collection, storage and transfer of data related to human biological material in the context of research projects entails the risk of breach of confidentiality (e.g. the possibility of identifying the donor), in particular, with regard to genetic information even if it is anonymized. Therefore, the WG-GMEC "broad consent" template clearly states that these risks cannot be completely excluded, and that they increase with increasing amounts of linked data, particularly, when additional genetic information is made available by the donor/patient himself, e.g. in the internet for purposes of genealogy [23]. Human biobanks and/or associated data warehouses shall take all appropriate measures 
according to the current state of technology to protect the privacy of their donors, including an implementation of novel anonymization tools using algorithms for controlled tampering/modification of research data (e.g. by systematically adding or transforming relevant parts of the anonymized data [37]).

\section{Data protection and pseudonymization}

As a general rule, personally identifiable information should never be transferred to a biobank but rather remain at the institution (hospital/treating physician) where the respective data were collected. By this procedure, personally identifiable information are protected by medical confidentiality and any access for third parties is regulated and/or restricted by criminal law [27].

\section{Pseudonymization of human biological material and related data}

In order to adequately secure the donors' privacy, it is generally recommended to store (or at least release) human biological material and related data in a double-pseudonymized (that is, double-coded) manner [28]. In addition, handling of pseudonymized data should generally involve a custodian (e.g. the independent data protection officer of the respective institution/university or a TTP); moreover, pseudonymized data should be hosted by at least two organizationally independent bodies (e.g. the pseudonymized data on the biospecimen by the biobank, and the related pseudonymized clinical patient data in a data warehouse of the hospital), each with separated responsibilities [38]. In case personally identifiable information are not or no longer required for accomplishing the scientific/research aims, data should be anonymized, unless conflicting with donor's rights or legal requirements [21, 28].

\section{Pseudonymization for release and use of biological material and related data}

As a general rule, for (cross-border) medical research projects, biological material and related data shall always be released and used in an at least doublecoded/pseudonymized manner. The donor pseudonyms employed when (cross-border) transferring biological material and/or related data must be different from those employed internally. This is accomplished by an additional pseudonymization procedure, carried out each time when samples and data are transferred, by which the internal donor pseudonyms are substituted for new, unrelated pseudonyms. Thus, any attempt to re-identify donors from samples or data, particularly by combining several requests for samples and data, is rendered almost impossible [19, 25, 28].

Bound by material transfer or data access agreements (material transfer agreement/data access agreement [MTA/DAA]) researchers must be kept from any attempt of identifying individuals, from whom biological material and/or person-related data have been received and/or used. Biological material and related data must be released only to projects and institutions (countries) applying appropriate data protection safeguards. The transfer of human biological material and/or related data to non-European Union (EU)/non-European Economic Area (EEA) countries may only take place, if the respective third country ensures a level of measures and procedures to protect privacy that adheres to current EU regulations (Regulation EU 2016/679 [39]). Today, with the implementation of the European General Data Protection Regulation (GDPR), an explicit "opt-in” for any transfer of biological material and/or related data in countries not fulfilling the GDPR criteria (as judged by the EU commission) is mandatory and should be integrated in the respective consent forms. If the country concerned fails to ensure sufficient privacy protection, biological material and/or related data must be anonymized prior to transfer. In any case, the pseudonymization keys must remain in the EU $[8,19,28]$.

\section{Reversal of double pseudonymization/ conditions of de-identification}

Any de-identification of pseudonymized biological material and/or person-related data is only permitted (i) if safety and/or security issues of donors (including treatable life-threatening unsolicited findings) or (ii) if proper completion of or safety issues related to a clinical research project (e.g. follow-up studies) are at stake [3, $6,8,9,28]$.

The WP-GMEC "broad consent" template clearly states that the donor/patient cannot expect any direct personal advantage or health benefit from his/her donation of biological material and related data. The results obtained will exclusively serve for medical research purposes. However, a feedback of research results is possible in case of a 
considerable health risk for the donor/patient that can be eliminated or lowered (e.g. if a potentially life-threatening disease can be avoided or treated - see also "Conditions for donor re-contact"), though the donor/patient must be pointed to the fact that he/she may be obliged to disclose this information to a third party (e.g. when applying for health or life insurances). On the other hand - as a basic principle - donors/patients must retain the possibility of refusing re-contact by simply ticking a corresponding box in the consent form (for exceptions see "Conditions for donor re-contact").

For the researchers, however, (double) pseudonymization must be safeguarded under all circumstances $[8,9$, 28]. Biobank staff has to be bound by professional discretion in case they do not underlie medical confidentiality. As a general rule, de-identifying pseudonymized data or re-contacting a donor should invariably involve a custodian (e.g. the independent data protection officer of the respective institution/university or a TTP). Anyway, as a measure of transparency, according to the new EU-GDPR, the function, responsibilities and contact information of the data protecting bodies are now to be detailed in the donor/patient information (e.g. the institutional data protection officer ["local custodian"] and the data protection officer of the respective state, where the institution is localized).

\section{Duration of storage and the right to withdraw consent}

Dependent on the respective consent, for medical research purposes human biological material and related data may be stored either for a limited time period, which may range from decades to a century, but also for unlimited time $[3,5,6,8,21,23,28,36]$. It is essential that donors may withdraw consent (in oral or written form) at any time without justification in an informal, low threshold way, and without any reprisal. Withdrawal must be documented by the body/person/institution receiving this information. Upon withdrawal of consent - as a general rule - the donor's biological material and related data must either be deleted or anonymized (unless the donor explicitly permits further use of his/her biological material and/or related data in a pseudonymized fashion). If the donor requests destruction of his/her biological material and deletion of all related data, the pseudonymization key must be deleted only after destruction of the biological material [28]. However, data cannot be removed from completed studies/analyses and/or published research results $[23,28,36]$.

\section{Use and release of biological material and data for cross-border research projects}

For any release of human biological material (local, national or cross-border), it must be ensured that any misuse of biological samples and/or person-related data is impossible; in addition, transparent access rules for researchers to both human biological material and the related data must have been implemented, and this implementation should be acknowledged and controlled [8, 19, 21, 23, 28, 36].

The user/handling guidelines of a human biobank should ensure that, prior to any release/transfer and use of human biological material and/or related data, approval of the intended research project by an independent competent ethics committee has been obtained. If the applicant fails to furnish any approval, sufficient justification must be provided why this may be not required or not appropriate in this particular case [28, 29].

For example, dependent on the type and scope of the intended research project (and dependent on the respective national law), the involvement of access committees instead of independent competent ethics committees might be sufficient $[7,8,33]$ (examples for exemptions in the German context have been given above, see Approval of applying research projects).

In any event, the biobank finally decides if and to what extent a transfer and use of human biological material and/or related data will be granted. The access and prioritization rules of a human biobank should be stipulated transparently in the by-laws and/or user guidelines. These rules should be submitted along with the documents assessed by the local independent competent ethics committee, as handled by most German university hospital-associated (human) biobanks [8, 28, 29].

Prior to any release/transfer of human biological material and/or related data, the biobank has to secure that the consent by the respective donor is valid and fully covers the purpose of use described in the intended research project [10-12].

Together with the release/transfer of human biological material and/or related data, the applicants' rights of use should be time-limited, project-specific and nontransferable. Duplication or transfer of human biological material and/or data to third parties is only permitted if this is clearly indicated in the proposal, indispensable to accomplish the intended research aim(s), and compliant with the MTA of the respective biobank.

Obligations of the user(s), in particular the obligation to secure the donor's rights and privacy, should be laid down in a written contract; this also includes the future publication of research results, in particular the publication of 
individual genetic information [23, 28, 36]. In addition, it must be secured that upon withdrawal the donor's nonanonymized biological samples will generally be discarded; however, with the explicit permission of the respective donor(s), his/her (their) remaining biological samples may be further used in an anonymized (or even pseudonymized) fashion. Human biological material that has not been used should either be returned to the biobank that has released the material or destroyed in mutual accordance [28].

\section{Conditions for donor re-contact}

The user/handling guidelines of a human biobank should clearly determine if and under which conditions/circumstances re-contact of individual donors is permitted. For researchers, however, pseudonymization must not be disclosed on any account [28].

The user/handling guidelines of a biobank as well as the information document for potential donors should, in particular, define under which conditions and by which process incidental or unsolicited findings will be returned to the donor(s), e.g. if the onset of a potentially life-threatening disease can be prevented or a (possibly not yet diagnosed) life-threatening disease can be treated [28]. In general, any de-pseudonymization or re-contacting of a donor should involve a custodian (e.g. the independent data protection officer of the respective institution/university or a TTP) and subsequently, on a case-to-case basis, the information and consultation by an appropriately qualified physician and/or human geneticist. In doing so, the donor's fundamental right of "not to know" (which is operationalized by an "opt-in" option in the consent form) must generally be respected. However, obligations that might result from professional law (for example, in case of emergency, or in case of incidental findings requiring urgent intervention, e.g. discovery of a previously undetected aneurysm of the ascending aortic arch with a high risk for rupture) remain unaffected $[8,28,31]$.

In addition, a human biobank should evaluate the efficiency of the implemented feedback procedure(s) (e.g. evaluate the appropriateness of the number and frequency of feedbacks given per year) on a regular basis.

\section{Quality management and control, transparency and public outreach}

The juridical owner (that is, the legally responsible institution/body, e.g. the university or the university hospital) as well as the head of a biobank must ensure compliance with all procedures and processes; compliance must be documented according to current standards/guidelines [20, 25, 26, 32]. Biobank staff must be trained for their specific tasks and must be bound by professional discretion $[8,21,28]$.

Larger (e.g. interdisciplinary and/or faculty-wide operating or cross-project) biobanks should regularly inform the public on their activities, e.g. by releasing information and research results on their homepage and/ or an easily accessible appropriate web-portal [28]. These tools should be addressed as an important part of the governance and communication strategy of each individual (clinical) biobank which should always have a focus on "stakeholder management" and, in particular, should foresee and implement web-based strategies of how to give feedback on (general) research results to donors/ patients, and how to receive (individual) feedback from donors/patients (e.g. interactive donor/ patient site, discussion corner, responses to frequently asked questions [FAQs]). Communicating concepts, objectives and future benefits of biobanks for the general public is essential for biobanks in order to establish a trustful relationship with current and future donors [40-42].

The same holds true for all the other different internal (clinicians, clinician scientists, researcher, faculty, hospital department(s)) and external stakeholders (external user, government, founders, press) of a biobank, requiring regular stakeholder analysis together with an implementation of strategies and tools to assess and to adequately respond to the respective stakeholder needs [34, 35, 43].

\section{Conclusions}

Nowadays, the openness of future medical questions and future challenges for public health besides specific research projects also requires broad, including crossborder, use of human biological material and related data. There is international $[3,4,6,9]$ and EU-wide consensus $[5,7,8]$ that broad consent is ethically legitimate based on two key arguments: (i) opening new vistas for medical research and (ii) optimizing public health care $[6,8,21,24$, 28]. However, in addition to public involvement and transparency of all governance processes of human biobanks striving for "broad consent" biospecimen collections, as a pre-condition for the legal validity of a donor's broad consent, the unpredictability of the future use of donated biological material and related data must be compensated by appropriate procedures $[6,8,21,22,25]$.

Thus, in Germany, involving independent competent ethics committees is necessary for both an assessment of 
human research biobanks themselves and (especially if legally required) an assessment of individual research projects making use of "broad consent" biological samples [28, 29]. Potential donors must be informed unmistakably about the broad scope of the intended use of his/her biological material and related data including the option of crossborder medical research $[8,19]$. In this regard, we have learned much from previous population-based studies and disease-specific comprehensive national research networks, including their strategies for donor's/study participant's consent - and feedback management as well as stakeholder-oriented governance strategies, including access and sharing rules for human biological material and related data [10-12]. Experience gained from those previous and current nation-wide endeavors [15] strongly suggests that even under "broad consent" conditions a donor should be permitted to exclude certain research fields and/ or procedures from the intended use of his/her biological material and related data, at least to some extent (similar but not identical to a so-called dynamic consent [30]).

In addition, donors must have the unconditional right to withdraw their consent and to have their biological material destroyed in that case.

Last but not least, human research biobanks are strongly recommended to reciprocate by making their implemented rules (governance/policies) and processes transparent and by fostering public involvement. Communicating concepts, objectives, results and future benefits of biobanks for both the respective donors and the general public is mandatory for human research biobanks in order to establish a trustful relationship with current and future donors [40-43].

Acknowledgments: Important elements of the present paper were compiled by the Working Group "Biobanking" of the Working Party of the German Medical Ethics Committees (http//www.ak-med-ethik-komm.de/index. php?option $=$ com_content\&view $=$ article\&id $=37$ :biobanke n\&catid=11\&Itemid=116\&lang=de) and orally presented at the European Biobank Week 2016, Vienna, Austria.

Author contributions: All the authors have accepted responsibility for the entire content of this submitted manuscript and approved submission.

Research funding: RJ receives funding from the BMBF, Grant FKZ 01EY1712 and from the IZKF Würzburg, Project Z9.

Employment or leadership: None declared.

Honorarium: None declared.

Competing interests: The funding organization(s) played no role in the study design; in the collection, analysis, and interpretation of data; in the writing of the report; or in the decision to submit the report for publication.

\section{References}

1. Pigeot I, Hummel M. Biobanken - eine entscheidende Ressource für erfolgreiche biomedizinische Forschung. Bundesgesundheitsblatt - Gesundheitsforschung - Gesundheitsschutz 2016;59:301-3.

2. Taupitz J, Weigel J. Biobanken - das Regelungskonzept des Deutschen Ethikrates. Wissenschaftsrecht 2012;45:35-81.

3. Hansson MG, Dillner J, Bartram CR, Carlson JA, Helgesson G. Should donors be allowed to give broad consent to future biobank research? Lancet Oncol 2006;7:266-9.

4. Caulfield T, Kaye J. Broad consent in biobanking: reflections on seemingly insurmountable dilemmas. Med Law Int 2009;10:85-100.

5. Thompson R, Johnston L, Taruscio D, Monaco L, Béroud C, Gut IG, et al. RD-connect: an integrated platform connecting databases, registries, biobanks and clinical bioinformatics for rare disease research. J Gen Intern Med 2014;29:780-7.

6. Grady C, Eckstein L, Berkman B, Brock D, Cook-Deegan R, Fullerton SM, et al. Broad consent for research with biological samples: workshop conclusions. Am J Bioeth 2015;15:34-42.

7. Strech D, Kahrass H, Hirschberg I. Research guideline recommendations for broad consent forms in biobank research and how they are currently addressed in practice. Am J Bioeth. 2015;15:60-3.

8. Jahns R. Establishing and operating a human biobank. Ethical aspects. Bundesgesundheitsblatt-GesundheitsforschungGesundheitsschutz 2016;59:311-6.

9. Bledsoe MJ. Ethical legal and social issues of biobanking: past, present, and future. Biopreserv Biobank 2017;15:142-7.

10. Wichmann H-E, Gieger C, Illig T. KORA-gen - resource for population genetics, controls and a broad spectrum of disease phenotypes. Gesundheitswesen 2005;67(S 01):26-30.

11. Völzke H, Alte D, Schmidt CO, Radke D, Lorbeer R, Friedrich $\mathrm{N}$, et al. Cohort profile: the study of health in pomerania. Int J Epidemiol 2011;40:294-307.

12. Schneider A, Rückerl R, Standl M, Markevych I, Hoffmann B, Moebus S, et al. Epidemiologische Studien mit Umweltbezug in Deutschland. Bundesgesundheitsblatt - Gesundheitsforschung - Gesundheitsschutz 2018;61:697-709.

13. Langanke M, Brothers KB, Erdmann P, Weinert J, Krafczyk-Korth J, Dörr M, et al. Comparing different scientific approaches to personalized medicine: research ethics and privacy protection. Pers Med 2011;8:437-44.

14. NAKO - German National Cohort [Internet]. NAKO [cited 2018 Jul 31]. Available from: https://nako.de/informationen-auf-englisch/.

15. Grabe HJ, Assel H, Bahls T, Dörr M, Endlich K, Endlich N, et al. Cohort profile: Greifswald approach to individualized medicine (GANI_MED). J Transl Med 2014;12:144.

16. DKTK - German Cancer Research Center [Internet]. [cited 2018 Jul 31]. Available from: https://dktk.dkfz.de/en/home.

17. Deutsches Zentrum für Lungenforschung (DZL e.V.) [Internet]. [cited 2018 Jul 31]. Available from: https://www.dzl.de/index. php/en/.

18. German Centre for Cardiovascular Research: DZHK [Internet]. [cited 2018 Jul 31]. Available from: https://dzhk.de/en/.

19. Hansson MG, Dahlin MK, Howard HC. BBMRI ELSI Workshop Report: sharing and access to data and human biospecimens for the benefit of patients [Internet]. BBMRI ELSI Workshop Report. 2015. Available from: http://www.bbmri-eric.eu/wp-content/ 
uploads/2016/08/FINAL-BBMRI-Paris-2015-WORKSHOPREPORT.pdf.

20. WMA. WMA Declaration of Taipei on Ethical Considerations regarding Health Databases and Biobanks [Internet]. Available from: https://www.wma.net/policies-post/wma-declaration-oftaipei-on-ethical-considerations-regarding-health-databasesand-biobanks/.

21. Taupitz J, Schreiber M. Biobanken - zwischen Forschungs- und Spenderinteressen. Bundesgesundheitsblatt - Gesundheitsforschung - Gesundheitsschutz 2016;59:304-10.

22. Strech D, Bein S, Brumhard M, Eisenmenger W, Glinicke C, Herbst $\mathrm{T}$, et al. A template for broad consent in biobank research. Results and explanation of an evidence and consensus-based development process. Eur J Med Genet 2016;59:295-309.

23. Arbeitskreis medizinischer Ethik-Kommissionen in Deutschland. Mustertext zur Information und Einwilligung in die Verwendung von Biomaterialien und zugehöriger Daten in Biobanken [Internet]. 2016 [cited 2017 May 19]. Available from: http://www. ak-med-ethik-komm.de/docs/MustertextBiobanken.docx.

24. Hirschberg I, Kahrass H, Strech D. International requirements for consent in biobank research: qualitative review of research guidelines. J Med Genet 2014;51:773-81.

25. Committee of Ministers to member States. Recommendation CM/Rec(2016)6 of the Committee of Ministers to member States on research on biological materials of human origin [Internet]. Recommendation CM/Rec(2016)6 of the Committee of Ministers to Member States on Research on Biological Materials of Human Origin; 2016 [cited 2017 May 19]. Available from: https://search. coe.int/cm/Pages/result_details.aspx?Objectld=09000016806 $4 \mathrm{e} 8 \mathrm{ff}$.

26. OECD. OECD Guidelines on Human Biobanks and Genetic Research Databases [Internet]; 2009 [cited 2017 May 19]. Available from: https://www.oecd.org/sti/biotech/44054609.pdf.

27. Andorno R, Gennet E, Jongsma K, Elger B. Integrating advance research directives into the European Legal Framework. Eur J Health Law 2016;23:158-73.

28. Arbeitskreis medizinischer Ethik-Kommissionen in Deutschland. Recommendation for the Assessment of Research-Related Human Biobanks by Ethics Committees [Internet]. 2016 [cited 2017 May 23]. Available from: http://www.ak-med-ethik-komm. de/docs/Recommendations2016_draft2016_09_07.pdf.

29. Taupitz J. The Role of Ethics Committees in Research with Materials of Biobanks. In: Nationales Biobanken-Symposium, Hummel $M$, editors. Biobanken-Forschung in Deutschland: Vom Konzept zur Realisierung: Tagungsband des 3 Nationalen BiobankenSymposiums vom 3-4 Dezember 2014 in Berlin. Berlin: AKA, 2014:125-33.

30. Kaye J, Whitley EA, Lund D, Morrison M, Teare H, Melham K. Dynamic consent: a patient interface for twenty-first century research networks. Eur J Hum Genet 2014.

31. Bundesärztekammer. (Muster-)Berufsordnung für die in Deutschland tätigen Ärztinnen und Ärzte (Stand 2015) [Internet]. Bundesärztekammer [cited 2017 May 23]. Available from: http:// www.bundesaerztekammer.de/recht/berufsrecht/muster-berufsordnung-aerzte/muster-berufsordnung/.

32. WMA. World Medical Association Declaration of Helsinki: ethical principles for medical research involving human subjects. J Am Med Assoc 2013;310:2191-4.
33. Strech D. Ethical review of biobank research: should RECs review each release of material from biobanks operating under an already-approved broad consent and data protection model? Eur J Med Genet 2015;58:545-9.

34. Simon CM, L'Heureux J, Murray JC, Winokur P, Weiner G, Newbury $\mathrm{E}$, et al. Active choice but not too active: public perspectives on biobank consent models. Genet Med 2011;13:821-31.

35. Richter G, Krawczak M, Lieb W, Wolff L, Schreiber S, Buyx A. Broad consent for health care-embedded biobanking: understanding and reasons to donate in a large patient sample. Genet Med 2018;20:76-82.

36. Arbeitskreis medizinischer Ethik-Kommissionen in Deutschland. Template For informed consent concerning the donation, storage, and utilization of biological materials as well as collecting, processing, and usage of (related) data in biobanks [Internet]. 2015 [cited 2017 May 17]. Available from: http://www.ak-med-ethik-komm.de/docs/Template-forinformed-consent.docx.

37. Sariyar M, Schlünder I. Reconsidering anonymization-related concepts and the term "identification" against the backdrop of the European legal framework. Biopreservation Biobanking [Internet]. 2016 Apr 22 [cited 2016 Apr 25]. Available from: http://online.liebertpub.com/doi/full/10.1089/bio.2015.0100.

38. Pommerening K, Müller T. Leitfaden zum Datenschutz in medizinischen Forschungsprojekten: generische Lösungen der TMF 2.0. Berlin: MWV, Med. Wiss. Verl.-Ges, 2014:260. (Schriftenreihe der TMF - Technologie- und Methodenplattform für die Vernetzte Medizinische Forschung e.V).

39. Regulation (EU) 2016/679 of the European Parliament and of the Council of 27 April 2016 on the protection of natural persons with regard to the processing of personal data and on the free movement of such data, and repealing Directive 95/46/EC (General Data Protection Regulation) [Internet]. Available from: http://ec.europa.eu/justice/data-protection/reform/files/regulation_oj_en.pdf.

40. Gaskell G, Gottweis H. Biobanks need publicity. Nature 2011;471:159-60.

41. Lander J, Hainz T, Hirschberg I, Strech D. Current practice of public involvement activities in biomedical research and innovation: a systematic qualitative review. Virolle M-J, editor. PLoS One 2014;9:e113274.

42. Lesch W, Schütt A, Jahns R. Biobanken in der öffentlichen Wahrnehmung: Verständnis, Interesse und Motivation von Probenspendern in Deutschland. In: Lesch W, Anhäuser M, Schütt A, editors. Gesundheitsforschung kommunizieren, Stakeholder Engagement gestalten: Grundlagen, Praxistipps und Trends. Berlin: Medizinisch Wissenschaftliche Verlagsgesellschaft, 2016:113-21 (Schriftenreihe der TMF - Technologie- und Methodenplattform für die Vernetzte Medizinische Forschung e.V.).

43. Lesch W, Schütt A, Jahns R. Was erwarten Wissenschaftler von zentralisierten Biobanken? Eine qualitative Stakeholder-Analyse. In: Lesch W, Anhäuser M, Schütt A, editors. Gesundheitsforschung kommunizieren, Stakeholder Engagement gestalten: Grundlagen, Praxistipps und Trends. Berlin: Medizinisch Wissenschaftliche Verlagsgesellschaft, 2016:161-73 (Schriftenreihe der TMF - Technologie- und Methodenplattform für die Vernetzte Medizinische Forschung e.V.). 